\title{
Fractional Quantum Hall from Overlap of Electron-Hole Bands
}

\author{
Fractional Excitonic Insulator \\ Authors: Yichen Hu, Jrn W. F. Venderbos, C. L. Kane \\ arXiv: 1806.05681 \\ Phys. Rev. Lett. 121, 126601 (2018) \\ Recommended with a Commentary by Ashvin Vishwanath, \\ Harvard University
}

Despite three decades of study, the fractional quantum Hall (FQH) effect remains one of the most remarkable phenomena in condensed matter physics. In addition to the quantization of Hall conductance to fractions of $e^{2} / h$, FQH states realizes quasiparticle excitations that carry fractional charge and anyon statistics. Ultimately, all these phenomena arise from the breakup or fractionalization of the electron. Obtaining such an unusual form of matter requires rather special conditions. Indeed the traditional route to realizing FQH states involves restricting electrons to two dimensions in a strong magnetic field when the spectrum consists of degenerate Landau levels. Further, on partially filling a Landau level and cooling to low temperatures, FQH states emerge - for example the celebrated Laughlin state emerges at $1 / 3$ filling of the lowest Landau level.

Clearly, it would be desirable to find other, less restrictive settings where the FQH effect could occur. Fractional Chern insulators (FCIs) have been proposed to occur in crystals with 'Chern bands', two dimensional band structures which carry a Chern number, implying a topology similar to Landau levels. On partially filling such Chern bands and in the presence of strong electron-electron interactions, FQH states have been shown, in theory, to emerge. Although this situation is conceptually rather similar to partially filling a Landau level, it may confer practical advantages in realizing FQH at elevated temperatures.

In contrast, the featured reference points out an entirely new regime for the FQH.

The proposed ingredients are: (i) a two dimensional crystal with broken time reversal (and reflection) symmetry. No magnetic field is applied. This ingredient is common to the previously mentioned fractional Chern insulators. However the following two ingredients are new. (ii) The electron filling is an integer and not a fraction and (iii) An overlap of two bands, an electron band and a hole band, with different angular momenta. It is argued that when the difference in angular momentum between the two bands $m$ is odd, a quantum Hall state can emerge. It is readily shown that an integer quantum Hall state will occur when $m= \pm 1$, even for free electrons. However, the nontrivial claim is that FQH states can arise when $|m|>1$ and an odd integer. Note, in this setup $m$ also indexes the Laughlin state realized, which is controlled, not by the fractional filling, but by the difference in angular 
momentum of the two touching bands. The quantized Hall conductance is predicted to be $1 / m$ in units of $e^{2} / h$, despite the integer filling.

To contrast this with the usual setup, recall that in the case of the lowest Landau level, the Laughlin states take the form:

$$
\Psi\left(z_{1}, z_{2}, \ldots, z_{N}\right)=\prod_{i<j}\left(z_{i}-z_{j}\right)^{m} e^{-\sum_{i}\left|z_{i}\right|^{2} / 4 l_{B}^{2}}
$$

where $l_{B}$ is the magnetic length and $m$ is an odd integer. The ' $\mathrm{j}$ 'th particle is labeled by the complex coordinates: $z_{j}=x_{j}+i y_{j}$. When $m=1$, this is the Integer quantum hall effect, which is nothing but the Slater determinant wavefunction of noninteracting electrons obtained by filling all states in the Landau level. However, when $m=3$ this gives the Laughlin state with fractional Hall conductance 1/3. A key feature is the presence of 'vortices', or zeros of the wavefunction, the factors $\left(z_{i}-z_{j}\right)^{3}$ in the wavefunction. The higher the power involved, the more effectively the electrons are kept apart, which forms the intuitive basis for the stability of the FQH state in the presence of repulsive interactions.

In the featured reference, both an electron and a hole band is present, and the locations of the electrons $z_{j}$ and holes $w_{j}$ are introduced in the many body wave-function (see also Ref.[1]). One is in the 'compensated' regime, with equal numbers of electrons and holes. Consider now a wave-function in which the amplitude for a configuration with $N$ electrons and $N$ holes is given by:

$$
\Psi^{N}\left(\left\{z_{j}, w_{j}\right\}\right)=\frac{\prod_{i<i^{\prime}}\left(z_{i}-z_{i^{\prime}}\right)^{m} \prod\left(w_{j}-w_{j^{\prime}}\right)^{m}}{\prod_{i, j}\left(z_{i}-w_{j}\right)^{m}}
$$

It is argued that under certain assumptions this is in the same topological phase as the Laughlin $m$ state. In particular it is easily shown that the $m=1$ is simply the ground state of non-interacting electrons forming a Chern insulator, when a pair of bands that differ by angular momentum 1, overlap. A model that demonstrates this is:

$$
H_{m=1}=\sum_{k} \epsilon(k)\left[c_{k}^{\dagger} c_{k}-d_{k}^{\dagger} d_{k}\right]+\Delta_{k} c_{k}^{\dagger} d_{k}+\text { h.c. }
$$

where the kinetic energy $\epsilon(k)=k^{2} / 2-v$ is opposite for the two bands, the electrons operators being $c$ for the electron band and $d$ for the hole band. Generally these bands will mix, which is captured by the second term, the mixing matrix element $\Delta$ has to account for the difference in angular momentum of the two bands. When the angular momentum of the electron and hole bands differs by 1 , then $\Delta_{k} \sim\left(k_{x}+i k_{y}\right)$. Now, as a function of the band overlap, which is controlled by $v$, one gets different phases (see Figure). When $v<0$, the bands are separated and one has a trivial insulator. But as we push the bands on top of one another by increasing $v$, the band gap vanishes at $v=0$. Further for $v>0$ one obtains a 'band inversion' and the gap reopens to give an insulator. It can be shown that this is an integer quantum hall (or Chern) insulator and the resulting wavefunction has the form in Eqn1 with $m=1$.

The intellectual leap made in the paper is for the case when $m \neq \pm 1$ but an odd integer. Now, the band overlap function is $\Delta_{k} \sim\left(k_{x}+i k_{y}\right)^{m}$. Again, for noninteracting electrons a similar logic would predict a transition from a regular insulator to an insulator with integer 


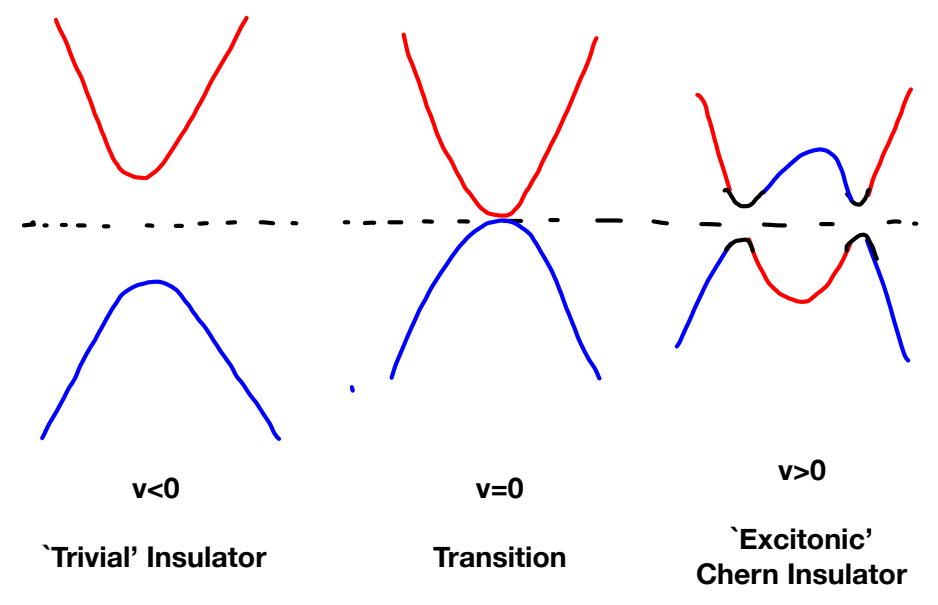

Figure 1: Phase Diagram for electron and hole bands with different angular momenta as a function of band overlap ' $v$ '.

Chern number (albiet different from unity). However, in the presence of repulsive electronelectron interactions, it is noted that the wave-function in Eqn. 1 does a much better job of keeping electrons away from other electrons (and keeping holes away from other holes). One may then expect a transition into a FQH state in the presence of interactions, if the conditions are right. Indeed the featured reference constructs toy models with interactions that are tailored to obtain ground states that include the state given by Eqn. 1. It remains to be seen if more realistic models will also lead to a FQH ground state, and more importantly if they lead to a realization in experiments.

If this program is successful, it will help realize the long standing hope of obtaining exotic quantum states in relatively mundane electronic systems.

I thank Eslam Khalaf for useful discussions.

\section{References}

[1] J. Dubail and N. Read Phys. Rev. B 92, 205307 (2015). 